\title{
Protocolos de medicação pré e pós-operatória para cobertura radicular combinada com enxerto de tecido conjuntivo.
}

\author{
Ana Paula Oliveira Giorgetti (1), Rafaela de Matos (1), Renato Corrêa Viana \\ Casarin (1), Suzana Prese Pimentel (1), Fabiano Ribeiro Cirano (1) e Fernanda \\ Vieira Ribeiro (1)
}

\section{ARTIGO ORIGINAL}

\section{Resumo}

Este estudo avaliou o efeito pré e pós-operatório de dexametasona e ibuprofeno na prevenção de dor / desconforto, edema e interferência na vida diária em pacientes submetidos à cobertura radicular combinada com enxerto de tecido conjuntivo subepitelial (CAF + CTG). Vinte pacientes foram aleatoriamente designados da seguinte forma: Grupo AINE: 400mg de ibuprofeno 60 min preemptivo + 400mg de ibuprofeno no pós-operatório; ou Grupo SAID: 4mg de dexametasona 60 min preemptiva $+4 \mathrm{mg}$ de dexametasona pós-operatória. A medicação pós-operatória foi administrada 8 e 16 horas após a cirurgia. Cada paciente recebeu questionários com base em uma escala numérica (escala numérica de 101 pontos [NRS-101]) e questões de múltipla escolha (escala de avaliação verbal de quatro pontos [VRS-4]) sobre dor / desconforto transoperatório, de hora em hora para $8 \mathrm{~h}$ após a cirurgia e uma vez por dia durante três dias. Também foi respondida a Escala Visual Analógica (EVA)

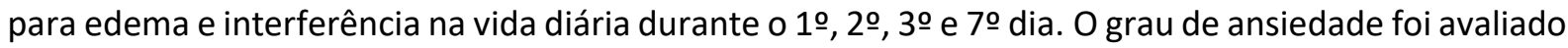
estatisticamente pelo teste Qui-quadrado. Os testes de Mann-Whitney e Friedman foram usados para os demais questionários. $O$ tempo de cirurgia e o número de comprimidos analgésicos consumidos foram comparados usando o teste $t$ de Student. Pacientes que fizeram uso de dexametasona apresentaram tendência a menos dor quando comparados aos indivíduos que ingeriram ibuprofeno, com diferença significativa observada três horas após o procedimento $(p<0,05)$. 0 uso de dexametasona também promoveu menos edema até o 2 o dia e menor interferência na vida diária no terceiro dia quando comparado ao ibuprofeno $(p<0,05)$.

Palavras-chave: antiinflamatório; drogas preventivas; medicamentos pós-operatórios; cirurgia periodontal; cobertura de raiz

Instituição afiliada: 1- UNIP - Universidade Paulista, São Paulo, SP, Brasil

Dados da publicação: Artigo recebido em 01 de agosto, revisado em 10 de agosto, aceito para publicação em 19 de agosto e publicado em 29 de Agosto.

DOI: https://doi.org/10.36557/2674-8169.2020v2n9p54-69

Fernanda Vieira Ribeiro ribeirofv@yahoo.com.br 


\section{Preemptive and Postoperative Medication Protocols for Root Coverage Combined with Connective Tissue Graft.}

This trial evaluated the preemptive and postoperative effect of dexamethasone and ibuprofen on prevention of pain/discomfort, edema and interference in daily life in patients undergoing root coverage combined with subepithelial connective tissue graft (CAF + CTG). Twenty patients were randomly assigned as follows: NSAID Group: 400mg Ibuprofen $60 \mathrm{~min}$ preemptive $+400 \mathrm{mg}$ Ibuprofen postoperative; or SAID Group: $4 \mathrm{mg}$ Dexamethasone $60 \mathrm{~min}$ preemptive $+4 \mathrm{mg}$ Dexamethasone postoperative. The postoperative medication was administered 8 and $16 \mathrm{~h}$ post-surgery. Each patient received questionnaires based on a numeric scale (101-point numeric scale rate [NRS-101]) and multiple choice questions (fourpoint verbal rating scale [VRS-4]) about trans-operative pain/discomfort, hourly for $8 \mathrm{~h}$ after surgery and once a day for three days. A Visual Analogue Scale (VAS) for edema and interference in daily life during the 1st, 2 nd, 3rd and 7th day was also answered. The degree of anxiety was rated statistically by the Chi-square test. The Mann-Whitney and Friedman tests were used for the other questionnaires. The surgery time and number of analgesic pills consumed were compared using Student's t-test. Patients who used dexamethasone presented a trend toward less pain when compared to individuals who ingested ibuprofen, with a significant difference observed $3 \mathrm{~h}$ after the procedure $(\mathrm{p}<0.05)$. The use of dexamethasone also promoted less edema until the 2 nd day and lower interference in daily life on the third day when compared with ibuprofen $(p<0.05)$. We concluded that the use of dexamethasone as a preemptive and postoperative medication was more suitable as a drug therapeutic protocol for CAF + CTG.

Key Words: anti-inflammatory; pre-emptive drugs; postoperative drugs; periodontal surgery; root coverage

Affiliated institution: 1- UNIP - Universidade Paulista, São Paulo, SP, Brazil

Publication data: Article received on August 1, revised on August 10, accepted for publication on August and published on August 29.

DOI: https://doi.org/10.36557/2674-8169.2020v2n9p54-69

Fernanda Vieira Ribeiro ribeirofv@yahoo.com.br 


\section{INTRODUÇÃO}

A recessão gengival é definida como o deslocamento da margem do tecido mole apical à junção cimento-esmalte ${ }^{1}$ e é uma característica clínica frequente em populações com bons ${ }^{2}$ ou maus ${ }^{3}$ padrões de higiene oral. Continua a ser um problema de alta prevalência ${ }^{4} \mathrm{e}$ tanto a estética quanto a hipersensibilidade dentinária são queixas recorrentes dos pacientes. Assim, os pacientes comumente perguntam sobre as opções de tratamento para defeitos de recessão bucal únicos e múltiplos.

Diversas abordagens cirúrgicas têm sido propostas para o tratamento dessas recessões. Dentre eles, o retalho coronariamente avançado (CAF) e o enxerto de tecido conjuntivo subepitelial são apresentados em metanálises ${ }^{5} \mathrm{e}$ várias revisões sistemáticas $6,7,8,9$ como tendo o maior potencial para redução da recessão e cobertura total da raiz ${ }^{6}$. Agudio et al. ${ }^{10}$, em estudo longitudinal (10 a 27 anos), mostraram que o tecido conjuntivo subepitelial do enxerto parece ser menos suscetível à recorrência da recessão gengival quando comparado às outras técnicas.

Embora a técnica de enxerto de tecido conjuntivo apresente boa previsibilidade e menores taxas de recorrência de recessão gengival, essa abordagem terapêutica requer um segundo sítio cirúrgico, geralmente o palato, como enxerto de sítio doador. Assim, essa técnica requer um tempo cirúrgico mais longo e pode causar dor, desconforto e edema no paciente, no trans e no pós-operatório. São ocorrências comuns após a cirurgia periodontal que podem surgir, principalmente nas primeiras $24 \mathrm{~h}^{11,12}$. Os medicamentos preventivos, com o objetivo de prevenir a dor e o desconforto pós-cirúrgico, são de grande importância. Diferentes medicamentos anti-inflamatórios administrados no pré-operatório, tanto anti-inflamatórios não esteroidais (AINEs) ou antiinflamatórios esteroides (SAIDs), demonstraram reduzir a intensidade da dor pós-operatória e a necessidade de analgésicos suplementares ${ }^{1,13}$. Os AINEs atuam bloqueando a via da ciclooxigenase (COX), produzindo efeitos no metabolismo do ácido araquidônico e na inibição da síntese de certos metabólitos, como as prostaglandinas (PGs) ${ }^{14}$. Os SAIDs, também definidos como corticosteroides, apresentam maior atividade do que os AINEs devido ao bloqueio da enzima fosfolipase $A 2$, reduzindo não só a liberação de PGs, mas também de outros mediadores químicos como leucotrienos e tromboxanos ${ }^{15}$, reduzindo o acúmulo de neutrófilos e justificando, pelo menos em parte, o poder dos anti-inflamatórios esteroides ${ }^{16}$.

Alguns resultados positivos têm sido mostrados com o uso de protocolos SAID e / ou AINE para controle da dor e edema pós-operatórios. Pilatti et al. ${ }^{17}$ avaliaram o uso preemptivo e pós-cirúrgico de $200 \mathrm{mg}$ de celecoxibe, um antiinflamatório não esteroidal (uma hora antes da cirurgia e $12 \mathrm{~h}$ após a primeira dose), e $4 \mathrm{mg}$ de dexametasona (uma hora antes da cirurgia e $8 \mathrm{~h}$ após a primeira dose) para o controle da dor pós-operatória após a cirurgia acesso para raspagem e alisamento radicular. Segundo os autores, tanto a dexametasona 
quanto o celecoxibe foram eficazes quando usados como medicação preemptiva e pósoperatória.

O efeito preemptivo de um inibidor não esteroidal de COX-2 com um SAID também foi demonstrado em cirurgias de alongamento de coroa, com ambos os protocolos de medicamentos antiinflamatórios apresentando um potencial semelhante para alívio de dor e edema após cirurgia periodontal ${ }^{18}$.

Embora alguns estudos tenham mostrado a vantagem do uso de medicamentos preemptivos no controle da dor e edema ao usar AINEs ou SAIDs, não há evidências conclusivas de ensaios clínicos sobre intervenções analgésicas preemptivas comparando ambos os protocolos de medicamentos após cobertura radicular combinada com tecido subepitelial enxertos. Com o objetivo de orientar e embasar cientificamente o processo de tomada de decisão dos protocolos medicamentosos propostos aos pacientes, o objetivo deste estudo foi avaliar o efeito da dexametasona e do ibuprofeno utilizados como medicamentos preemptivos em combinação com seu uso também no pós-operatório para prevenir dor / desconforto, edema e interferência no cotidiano após recobrimento radicular associado a enxerto subepitelial.

\section{MATERIAL E MÉTODOS}

\section{Design de estudo}

O desenho do estudo foi aprovado pelo Comitê de Ética da Universidade Paulista (Protocolo $\mathrm{n}$ - 142.019). Este estudo foi desenhado como um estudo paralelo randomizado duplo-cego para comparar dois medicamentos preemptivos diferentes em pacientes que requerem cobertura radicular combinada com enxerto subepitelial. Todos os pacientes receberam uma descrição detalhada do tratamento proposto e deram seu consentimento informado por escrito.

\section{Triagem de População}

Vinte pacientes selecionados na Clínica de Pós-graduação da UNIP foram submetidos a exames periodontais e radiográficos completos e entrevista clínica detalhada.

Os critérios de entrada da linha de base incluíram o seguinte: pelo menos um dente com recessão bucal $\geq 2 \mathrm{~mm}$ (classificado como Classe I ou II de Miller) ${ }^{19}$ que precisava ser submetido a cirurgia periodontal para cobertura radicular, pontuação de placa bucal total (FMPS) e total - pontuação de sangramento na boca (FMBS) $<20 \%$, vitalidade do dente e ausência de sulcos, irregularidades, cáries ou restaurações na área a ser tratada. 
Pacientes grávidas ou lactantes, com quaisquer condições que contra-indicassem o uso dos medicamentos do estudo ou que recebessem medicamentos foram excluídos do estudo. Os critérios de exclusão incluíram: gravidez, lactação, tabagismo atual ou tabagismo nos últimos 10 anos, condições sistêmicas (por exemplo, diabetes mellitus, condições instáveis ou com risco de vida) capazes de interferir no limiar de dor, analgésicos ou antiinflamatórios anteriores dois.

\section{Tratamento Pré-Cirúrgico}

Todos os pacientes tiveram um tratamento periodontal inicial que consistiu na remoção do cálculo supragengival por uma sessão de desbridamento e duas sessões de motivação com intervalo de 15 dias, durante as quais foram dadas instruções de higiene oral com escovas dentais padronizadas (Oral B Indicator Plus 30; Procter \& Gamble, São Paulo, SP, Brasil) e a técnica de Stillman em todos os pacientes.

Antes da cirurgia, as medidas clínicas foram reavaliadas pelo mesmo examinador calibrado (RM). Todos os parâmetros foram medidos com a sonda periodontal PCP-15 (Hu Friedy do Brasil, Rio de Janeiro, RJ, Brasil). O FMPS ${ }^{20}$ e o FMBS ${ }^{21}$ foram calculados após avaliação da presença de placa ou sangramento na sondagem do fundo da bolsa com sonda manual e cálculo da porcentagem de locais que revelaram a presença de placa ou sangramento. Posteriormente, aqueles que atenderam aos critérios de inclusão foram incluídos no estudo.

Os medicamentos preventivos foram administrados 60 minutos antes do procedimento cirúrgico oferecido pelo investigador do estudo antes do início da cirurgia. Grupo de inibidores AINE COX-1 e -2: doses únicas de 400 mg de ibuprofeno (Advil, Wyeth, Itapevi, SP, Brasil) ou Grupo SAID: doses únicas de $4 \mathrm{mg}$ de dexametasona (Decadron; Aché, Guarulhos, SP, Brasil). Os participantes não sabiam quais medicamentos estavam recebendo.

\section{Cálculo do tamanho da amostra}

O tamanho da amostra foi calculado usando $a=0,05$ e poder de $80 \%$. Para a variabilidade (s = DP), um valor de $10 \mathrm{~mm}$ foi usado usando os dados da escala NRS-101 (escala de taxa numérica de pontos) na dor / desconforto transoperatório como a variável de desfecho primário deste estudo. O valor mínimo clinicamente significativo (d) considerado foi de $15 \mathrm{~mm}$. Determinou-se que seria necessária uma amostra mínima de 7 pacientes por grupo. No entanto, considerando que alguns pacientes podem ser perdidos durante o acompanhamento, o número de indivíduos inscritos por grupo neste estudo foi 10 . Uma análise de poder post hoc deste estudo foi conduzida usando a variável de desfecho primário 
atingindo um valor de poder de 1,00 com o apresentar dados usando o poder de amostra do programa SPSS 21 (IBM, Armonk, NY, EUA).

\section{Procedimentos cirúrgicos}

Os procedimentos cirúrgicos foram realizados 30 dias após a terapia inicial. Antes da cirurgia, foi realizada antissepsia intraoral com solução de enxágue de clorexidina $0,12 \%$ e antissepsia extraoral com solução de iodo.

A anestesia local foi obtida com solução injetável de lidocaína a $2 \%$ com epinefrina 1: 100.000 (ALPHACAINE, DFL, Rio de Janeiro, Brasil); os pacientes foram tratados com o Retalho Coronally Advanced (CAF) combinado com enxerto de tecido conjuntivo subepitelial realizado pelo mesmo operador (MZC), diferente do examinador (RM). O procedimento cirúrgico foi descrito anteriormente ${ }^{8}$.

\section{Cuidados pós-operatórios}

No final da cirurgia, os pacientes de cada grupo receberam duas doses de medicamentos pós-operatórios, ou seja, 8 e 16 h pós-operatório, como segue: Grupo de inibidores de AINE COX-1 e -2: 400 mg de ibuprofeno ou Grupo SAID: 4 mg dexametasona. Os participantes não sabiam quais medicamentos estavam recebendo.

Todos os pacientes receberam três comprimidos de medicação analgésica (Paracetamol 750 mg, Medley, Campinas, SP, Brasil) e foram orientados a tomar a medicação a cada 8 horas, mas somente se sentissem dor. Os pacientes também foram orientados a quantificar a medicação analgésica tomada. Para o controle do biofilme, todos os pacientes, independente do tratamento recebido, foram orientados a enxaguar com solução de clorexidina $0,12 \%$ duas vezes ao dia durante 7 dias. As suturas foram removidas 7 dias após a cirurgia.

\section{Parâmetros avaliados}

A escala de ansiedade odontológica de Corah ${ }^{22}$ foi aplicada antes do procedimento cirúrgico devido ao fato de que o estresse e a ansiedade odontológica variam entre os pacientes e podem influenciar a percepção da dor do paciente. Os questionários foram baseados em escalas utilizadas anteriormente por Jensen et al. ${ }^{23}$, Peres et al. ${ }^{18}$ e Kolbe et al. ${ }^{24}$ uma escala de taxa numérica de 101 pontos (NRS-101) e questões de múltipla escolha (escala de classificação verbal de quatro pontos [VRS-4]), em que os pacientes responderam a perguntas sobre dor e desconforto de hora em hora nas primeiras 8 horas após a cirurgia e uma vez um dia por três dias. Além disso, por meio de uma escala visual analógica (EVA) 
Protocolos de medicação pré e pós-operatória para cobertura radicular combinada com enxerto de tecido conjuntivo.

Giorgetti et al.

horizontal de $100 \mathrm{~mm}$, os pacientes responderam questões relacionadas à percepção do edema e da interferência na vida diária promovida pelo procedimento cirúrgico no 1으, 2ㅇ, 3은 e 7o dia após a cirurgia.

\section{Análise Estatística}

O teste Qui-quadrado foi usado para avaliar estatisticamente o grau de ansiedade. Os testes de Mann-Whitney e Friedman foram usados para avaliação de outros questionários. $O$ tempo cirúrgico, o número de analgésicos ingeridos e a distribuição demográfica e as características dos dentes foram comparados pelo teste t de Student.

Um nível experimental de significância foi determinado em 5\% para todas as análises estatísticas $(a=0,05)$.

Este estudo é uma adaptação para o português do artigo original " Giorgetti, Ana Paula Oliveira, et al. "Preemptive and Postoperative Medication Protocols for Root Coverage Combined with Connective Tissue Graft." Brazilian dental journal 29.1 (2018): 23-29. 34 " E segue os preceitos Creative Commons - Atribuição 4.0 Internacional - CC BY 4.0 de livre distribuição, uso ou remixagem. Nenhum dos autores endossou este compartilhamento.

\section{RESULTADOS}

\section{Distribuição Demográfica e Características dos Dentes}

A caracterização do paciente e dos dentes está demonstrada na Tabela 1 e nenhuma diferença estatística ( $p>0,05)$ na análise intergrupos foi demonstrada mostrando homogeneidade de resultados para ambos os grupos.

Tabela 1: Características do paciente e dos dentes para ambos os grupos no início do

\begin{tabular}{lcc}
\hline Características & Grupo dexametasona & Grupo ibuprofeno \\
\hline Idade (anos; média \pm DP) & $44,30 \pm 12,60$ & $43,40 \pm 15,09$ \\
\hline Sexo $(\%$ feminino) & 60 & 70 \\
\hline Dentes molares $(\%)$ & 6,60 & 6,25 \\
\hline Dentes pré-molares $(\%)$ & 73,40 & 56,25 \\
\hline Dentes caninos $(\%)$ & 20 & 37,50 \\
\hline GR $($ mm; média \pm DP) & $3,80 \pm 1,48$ & $4,30 \pm 1,49$ \\
\hline
\end{tabular}

Nenhuma diferença significativa entre os grupos foi observada no início do estudo (teste t de Student; $p<0,05$ ); GR: recessão gengival. DP: desvio padrão.

estudo 


\section{Tempo de cirurgia e número de comprimidos analgésicos consumidos}

Em relação ao tempo cirúrgico necessário para o tratamento cirúrgico e ao número de comprimidos analgésicos consumidos no pós-operatório, não foram observadas diferenças estatisticamente significantes entre os grupos $(p>0,05)$ ( Tabela 2 ).

Tabela 2: Média ( $\pm \mathrm{DP}$ ) do tempo de cirurgia e do número de consumo de analgésico após a cirurgia no Grupo SAID (Dexametasona) e Grupo AINE (Ibuprofeno)

\begin{tabular}{lcc}
\hline Analgésico & Número de comprimidos consumidos (n) & Tempo de cirurgia (min) \\
\hline Dexametasona & $3,5 \pm 3,8 \mathrm{~A}$ & $135,0 \pm 35,2 \mathrm{~A}$ \\
\hline Ibuprofeno & $4,0 \pm 4,6 \mathrm{~A}$ & $110,0 \pm 44,7 \mathrm{~A}$ \\
\hline
\end{tabular}

SD: Desvio Padrão. Não foram observadas diferenças estatísticas entre os grupos (teste t de Student), $p>0,05$.

\section{Resultado dos parâmetros avaliados}

Em relação ao nível de ansiedade antes do procedimento cirúrgico, a maioria dos pacientes apresentou nível de ansiedade leve em ambos os grupos, sendo 7 pacientes no Grupo SAID e 5 no Grupo AINE. Representando o nível de ansiedade moderado, 3 pacientes pertenciam ao Grupo SAID e 2 pertenciam ao Grupo AINE. Embora nenhum dos pacientes do grupo SAID tenha demonstrado qualquer nível de ansiedade intensa, 3 deles o demonstraram no grupo AINE. No entanto, não foram observadas diferenças estatísticas entre os grupos para nenhum dos níveis de ansiedade $(p>0,05)$ ( Tabela 3 ).

Tabela 3: Número de pacientes para cada grupo [Grupo SAID (Dexametasona) e Grupo AINE (Ibuprofeno) quanto ao nível de ansiedade antes do procedimento cirúrgico.

\begin{tabular}{lcc}
\hline Nível / Droga & Dexametasona & Ibuprofeno \\
\hline Leve & 7 & 5 \\
\hline Moderado & 3 & 2 \\
\hline Intenso & 0 & 3 \\
\hline
\end{tabular}

Não houve diferenças estatisticamente significativas no nível de ansiedade entre os grupos (teste de MannWhitney; $p>0,05)$.

Com base na escala NRS, a intensidade da dor sentida durante o procedimento cirúrgico (momento trans-operatório) e no pós-operatório tardio foi estatisticamente semelhante entre os grupos SAID e AINE $(p>0,05)$, exceto após 3 pós-operatórios $h(3 h)$, quando o Grupo SAID apresentou nível de dor estatisticamente inferior $(p \leq 0,05)$ do que o Grupo AINE ( Tabela 4 ). Com relação à intensidade do desconforto ( Tabela 5), a maioria dos pacientes relatou intensidade 2 (com base no VRS de quatro pontos), em que apenas as intensidades 1 e 2 foram narradas, sem diferenças significativas observadas entre os grupos experimentais em qualquer um dos intervalos de tempo descritos $(p>0,05)$. Porém, para o Grupo AINH, apenas aos 3 dias de pós-operatório, foi relatado desconforto de intensidade 1 , 
ao contrário do Grupo SAID com 5 intervalos de tempo e mesma intensidade de nível de desconforto pós-operatório. Levando em consideração a intensidade do edema e a interferência na vida diária ( Tabela 6), ambos os grupos apresentaram redução ao longo do tempo e houve menor grau de edema após 7 dias de pós-operatório do que nos dias anteriores para os grupos SAID e AINE, principalmente na comparação do primeiro e do sétimo dia $(p \leq 0,05)$. Na análise intragrupo, no 2 o dia de pós-operatório, o grupo SAID apresentou edema estatisticamente significativo menos que o grupo AINE $(p<0,05)$.

Tabela 4: Média da intensidade da dor no transoperatório e pós-operatório tardio pela escala NRS-101 (escala de taxa numérica de pontos) no Grupo SAID (Dexametasona) e Grupo AINE (Ibuprofeno)

\begin{tabular}{lcc}
\hline Ponto de tempo & Dexametasona & Ibuprofeno \\
\hline Transoperatório & $24,0 \mathrm{Aa}$ & $11,0 \mathrm{Aa}$ \\
\hline $1 \mathrm{~h}$ & $18.5 \mathrm{Aa}$ & $18,5 \mathrm{Aa}$ \\
\hline $2 \mathrm{~h}$ & $10.5 \mathrm{Aa}$ & $13,5 \mathrm{Aa}$ \\
\hline $3 \mathrm{~h}$ & $5,5 \mathrm{Aa}$ & $19,3 \mathrm{Aa}$ \\
\hline $4 \mathrm{~h}$ & $8,5 \mathrm{Aa}$ & $16,3 \mathrm{Aa}$ \\
\hline $5 \mathrm{~h}$ & $3,5 \mathrm{Aa}$ & $14,3 \mathrm{Aa}$ \\
\hline $6 \mathrm{~h}$ & $3,8 \mathrm{Aa}$ & $9,3 \mathrm{Aa}$ \\
\hline $7 \mathrm{~h}$ & $3,2 \mathrm{Aa}$ & $8,3 \mathrm{Aa}$ \\
\hline $8 \mathrm{~h}$ & $2.7 \mathrm{Aa}$ & $7,5 \mathrm{Aa}$ \\
\hline 1 dia & $2.6 \mathrm{Aa}$ & $14,0 \mathrm{Aa}$ \\
\hline 2 dias & $3,5 \mathrm{Aa}$ & $19,5 \mathrm{Aa}$ \\
\hline 3 dias & $9,5 \mathrm{Aa}$ & $16,0 \mathrm{Aa}$ \\
\hline
\end{tabular}

Médias seguidas por diferentes letras maiúsculas nas linhas representam diferenças significativas entre os grupos pelo teste de Mann Whitney, $\mathrm{p}<0,05$. Médias seguidas por diferentes letras minúsculas nas colunas representam diferenças significativas intragrupo pelo teste de Friedman, $p<0,05$.

Tabela 5: Média da intensidade do desconforto transoperatório e pós-operatório tardio pela escala VRS-101 (escala de taxa verbal de quatro pontos) no Grupo SAID (Dexametasona) e Grupo AINE (Ibuprofeno).

\begin{tabular}{lll}
\hline Ponto de tempo & Dexametasona & Ibuprofeno \\
\hline Transoperatório & $2 \mathrm{Aa}$ & $2 \mathrm{Aa}$ \\
\hline $1 \mathrm{~h}$ & $2 \mathrm{Aa}$ & $2 \mathrm{Aa}$ \\
\hline $2 \mathrm{~h}$ & $2 \mathrm{Aa}$ & $2 \mathrm{Aa}$ \\
\hline $3 \mathrm{~h}$ & $2 \mathrm{Aa}$ & $2 \mathrm{Aa}$ \\
\hline $4 \mathrm{~h}$ & $2 \mathrm{Aa}$ & $2 \mathrm{Aa}$ \\
\hline $5 \mathrm{~h}$ & $1 \mathrm{Aa}$ & $2 \mathrm{Aa}$ \\
\hline $6 \mathrm{~h}$ & $1 \mathrm{Aa}$ & $2 \mathrm{Aa}$ \\
\hline $7 \mathrm{~h}$ & $1 \mathrm{Aa}$ & $2 \mathrm{Aa}$ \\
\hline $8 \mathrm{~h}$ & $2 \mathrm{Aa}$ & $2 \mathrm{Aa}$ \\
\hline 1 dia & $2 \mathrm{Aa}$ & $2 \mathrm{Aa}$ \\
\hline 2 dias & $1 \mathrm{Aa}$ & \\
\hline 3 dias & $1 \mathrm{Aa}$ & $\mathrm{Aa}$ \\
\hline
\end{tabular}

Médias seguidas por diferentes letras maiúsculas nas linhas representam diferenças significativas entre os grupos pelo teste de Mann Whitney, $\mathrm{p}<0,05$. Médias seguidas por diferentes letras minúsculas nas colunas representam diferenças significativas intragrupo pelo teste de Friedman, $p<0,05$. 
Tabela 6: Intensidade média (DP) do edema e interferência na vida diária no pósoperatório tardio pela escala VAS no Grupo SAID (Dexametasona) e Grupo AINE (Ibuprofeno).

\begin{tabular}{lccccc}
\hline Percepção & Medicamento & 1 dia & 2 dias & 3 dias & 4 dias \\
\hline \multirow{2}{*}{ Edema } & Dexametasona & $32,4 \pm 31,6 \mathrm{Aa}$ & $30,4 \pm 32,1 \mathrm{Ba}$ & $22,9 \pm 4,0 \mathrm{Aab}$ & $2,5 \pm 38,3 \mathrm{Ab}$ \\
\cline { 2 - 5 } & Ibuprofeno & $52,1 \pm 38,3 \mathrm{Aa}$ & $60,2 \pm 34,4 \mathrm{Aa}$ & $33,9 \pm 33,4 \mathrm{Aab}$ & $11,8 \pm 26,5 \mathrm{Ab}$ \\
\hline \multirow{2}{*}{ Interferência na vida diária } & Dexametasona & $34,5 \pm 28,5 \mathrm{Aa}$ & $29,4 \pm 28,2 \mathrm{Aa}$ & $15,4 \pm 25,7 \mathrm{Bab}$ & $2,4 \pm 4,6 \mathrm{Ab}$ \\
\cline { 2 - 6 } & Ibuprofeno & $53,0 \pm 35,0 \mathrm{Aa}$ & $44,2 \pm 35,9 \mathrm{Aa}$ & $35,3 \pm 33,6 \mathrm{Aab}$ & $6,8 \pm 7,9 \mathrm{Ab}$ \\
\hline
\end{tabular}

Médias seguidas por diferentes letras maiúsculas em uma linha representam diferenças significativas entre os grupos pelo teste de Mann Whitney, $p<0,05$. Médias seguidas de diferentes letras não maiúsculas na coluna representam diferenças significativas intragrupo pelo teste de Friedman, $p<0,05$.

\section{DISCUSSÃO}

A cirurgia periodontal para recobrimento radicular combinada com enxerto de tecido conjuntivo subepitelial tem se mostrado (6-8) a melhor opção para redução / resolução da recessão gengival, mas pode levar a maior dor e desconforto pós-operatório ${ }^{10}$, visto que são necessários dois sítios cirúrgicos. Para se obter um controle adequado da morbidade pósoperatória, a escolha dos protocolos de medicamentos é essencial. Nesse sentido, este é o primeiro estudo a comparar o desempenho preemptivo e pós-operatório de inibidores não esteroidais de COX-1 e COX-2 com antiinflamatório esteroidal para cobertura radicular combinada com enxertos de tecido conjuntivo subepitelial.

Independentemente dos esquemas farmacológicos, tanto o SAID quanto o AINE apresentaram resultado final positivo no controle dos sinais e sintomas inflamatórios no pósoperatório. No geral, os resultados deste estudo demonstram que o uso de dexametasona como medicação preemptiva e pós-operatória mostrou um efeito superior em comparação com ibuprofeno no controle da dor e desconforto após o tratamento. Baixas pontuações de dor foram registradas durante todo o período de avaliação, de 1 hora a 3 dias de pósoperatório para ambos os grupos. Assim, os antiinflamatórios preemptivos podem prevenir a hiperalgesia, reduzindo a dor e o desconforto pós-operatório e o consumo de analgésicos.

$\mathrm{Na}$ cirurgia de retalho mucoperiosteal para raspagem e alisamento radicular ${ }^{17}$ e no alongamento da coroa ${ }^{18}$, não foram demonstradas diferenças significativas para a intensidade da dor quando ambos os antiinflamatórios (SAID e AINE) foram comparados como medicamentos preemptivos e pós-operatórios. Steffens et al. ${ }^{25}$ também não mostraram diferença significativa para dor entre os grupos SAID ( $8 \mathrm{mg}$ de dexametasona) e AINE (120 mg de etoricoxibe); isso também foi verdadeiro para a cirurgia de retalho mucoperiosteal para raspagem e alisamento radicular, mas os medicamentos foram administrados apenas no período preemptivo. Por outro lado, Mehra et al. ${ }^{26}$ comparou diferentes regimes farmacológicos usando SAIDs e AINEs, e demonstrou que o AINE ibuprofeno produziu uma 
redução aumentada da dor. Os pacientes que receberam $600 \mathrm{mg}$ de ibuprofeno preventivo e pós-operatório por uma semana relataram maior eficácia na redução do nível de PGE 2 na urina e saliva em comparação com a dexametasona isolada após a remoção dos terceiros molares inferiores impactados. Além de utilizar os mesmos protocolos farmacológicos, as vias de administração dos medicamentos e a cirurgia de escolha nos estudos são diferentes, o que pode influenciar na morbidade das cirurgias.

No entanto, há uma tendência de redução dos níveis de dor com o uso de dexametasona. Resultados de pesquisas anteriores ${ }^{16,27}$ mostraram que o uso de SAID como medicação preemptiva foi eficaz na prevenção da dor após cirurgias de retirada de terceiros molares, o que está de acordo com o presente estudo, apesar da cirurgia realizada e da dosagem do medicamento mostrar melhor eficácia da dexametasona; isso pode ser explicado pelo mecanismo de ação dessa droga, uma vez que os corticosteroides inibem a enzima fosfolipase A2 reduzindo a liberação do ácido araquidônico em leucotrienos e células inflamatórias. Além disso, um menor acúmulo de neutrófilos pode justificar, pelo menos em parte, o poder dos SAIDs ${ }^{28}$. Por outro lado, os AINEs atuam bloqueando a via da ciclooxigenase, produzindo efeitos no metabolismo do ácido araquidônico e inibindo a síntese de alguns metabólitos como as prostaglandinas (PGs) ${ }^{29}$.

Além da dor, a morbidade da cirurgia também leva em consideração a intensidade do edema vivenciado no pós-operatório tardio, o que mostrou que o grupo que recebeu dexametasona como medicação preemptiva e pós-operatória apresentou menores taxas de edema quando comparado ao ibuprofeno. Alcântara et al. ${ }^{30}$, usando medidas lineares, demonstraram que a dexametasona controlou o edema melhor do que a metilprednisolona em todas as avaliações pós-operatórias. No entanto, de acordo com Peres et al. ${ }^{18}$, a intensidade do edema foi semelhante entre os grupos inibidor de COX-2 e SAID, o que está de acordo com Emery ${ }^{14}$. Embora as cirurgias de alongamento de coroa realizadas por Peres et al. ${ }^{18}$ causou baixo edema, seu acompanhamento foi de 4, 8, 12 e 24 horas após a cirurgia, o que é diferente do estudo atual, onde os pacientes foram analisados de hora em hora nas primeiras 8 horas após a cirurgia e uma vez por dia nos três dias subsequentes e o sétimo dia.

Ressalta-se que um aspecto importante deste estudo foi a avaliação da ansiedade do paciente antes da cirurgia por meio de uma escala de ansiedade ${ }^{22}$, considerando que o estresse e a ansiedade influenciam na percepção de dor / desconforto, pois esta é subjetiva e varia muito entre os indivíduos ${ }^{31,32}$. Outro aspecto importante do presente estudo está associado ao cuidado na avaliação do nível de ansiedade antes da cirurgia e no monitoramento do tempo cirúrgico necessário para os procedimentos e da quantidade de analgésicos consumidos no pós-operatório. Para todos esses aspectos avaliados, não foram observadas diferenças entre os grupos SAID e AINE, confirmando a homogeneidade dos grupos experimentais e apoiando os resultados obtidos no estudo. Essas avaliações não foram realizadas em outros estudos $(16,17,25-27,30)$, o que poderia afetar a interpretação dos dados de morbidade individual. 
Infelizmente, ambos os medicamentos apresentam efeitos colaterais adversos. O AINE, no caso o ibuprofeno, é responsável pela inibição da COX-1 e da COX-2, o que leva a alterações na mucosa gástrica e toxicidade renal. O uso clínico da dexametasona tem a vantagem de não atuar na via da ciclooxigenase, não promovendo alterações a nível gástrico e renal, mas seu uso deve ser moderado e racional, por tempo e dose limitados, pois, segundo os endocrinológicos análise, após 5 dias de uso, a terapia começa a produzir imunossupressão, podendo levar até nove meses para retornar aos níveis normais em alguns pacientes ${ }^{33}$. No entanto, como os medicamentos antiinflamatórios em intervenções cirúrgicas para cobertura radicular são necessários e prescritos apenas por curtos períodos de tempo, os benefícios promovidos por um protocolo de medicamento não esteroidal não teriam o impacto negativo que este medicamento promove quando utilizado por longos períodos de tempo, tornando a indicação deste protocolo segura e suportada do ponto de vista científico.

Dentro dos limites deste estudo, o presente estudo demonstrou que a dexametasona apresentou melhores resultados do que o ibuprofeno no controle da dor, edema e interferência na vida diária no pós-operatório para recobrimento radicular combinado com enxerto de tecido conjuntivo.

\section{REFERÊNCIAS}

1 American Academy of Periodontology. Glossary of Periodontal Terms, 3rd ed. Chicago: The American Academy of Periodontology, 1992. [ Links ]

2 Serino, G; Wennström, JL; Lindhe, J; Eneroth, L. The prevalence and distribution of gingival recession in subjects with a high standard of oral hygiene. J Clin Periodontol 1994;21:57-63.

[ Links ]

3 Löe, $\mathrm{H}$; Anerud, A; Boysen, $\mathrm{H}$. The natural history of periodontal disease in man: prevalence, severity, and extent of gingival recession. J Periodontol 1992:63:489-495. [ Links ]

4 Hugoson, A; Sjödin, B; Norderyd, O. Trends over 30 years, 1973-2003, in the prevalence and severity of periodontal disease. J Clin Periodontol2008;35:405-414. [ Links ]

5 Clauser, C; Nieri, M; Franceschi, D; Pagliaro, U; Pini Prato, GP. Evidence-based mucogingival therapy. Part 2: Ordinary and individual patient data meta-analyses of surgical treatment of recession using complete root coverage as the outcome variable. J Periodontol 2003;74:741756. [ Links ]

6 Cairo, F; Pagliaro, U; Nieri, M. Treatment of gingival recession with coronally advanced flap procedures: a systematic review. J Clin Periodontol 2008;35:136-162. [ Links ] 
7 Chambrone, L; Sukekava, F; Araújo, MG; Pustiglioni, FE; Chambrone, LA; Lima, LA. Rootcoverage procedures for the treatment of localised recession-type defects: a Cochrane systematic review. J Periodontol 2010;81:452-478. [ Links ]

8 Cortellini, P; Pini Prato, GP. Coronally advanced flap and combination therapy for root coverage. Clinical strategies based on scientific evidence and clinical experience.

Periodontology2000, 2012;59:158-184. [ Links ]

9 Chambrone, L; Tatakis, DN. Periodontal soft tissue root coverage procedures: a systematic review from the AAP Regeneration Workshop. J Periodontol 2015;86(2 Suppl):S8-S51.

[ Links ]

10 Agudio, G; Nieri, M; Rotundo, R; Franceschi, D; Cortellini, P; Pini Prato, GP. Periodontal conditions of sites treated with gingival-augmentation surgery compared to untreated contralateral homologous sites: a 10- to 27-year long-term study. J Periodontol 2009;80:1399-1405. [ Links ]

11 Silva, RCL; Riera, R; Saconato, H. Lumiracoxib for acute postoperative dental pain: a systematic review of randomised clinical trials. São Paulo Med J 2011;129:335-345. [ Links ]

12 Piecuch, JF. What strategies are helpful in the operative management of third molars? J Oral Maxillofac Surg 2012;70:s25-s32. [ Links ]

13 American Academy of Periodontology. Consensus report on mucogingival therapy. Proceedings of the World Workshop in Periodontics. Ann Periodontol 1996;1:702-706. [ Links ]

14 Emery, P. Considerations for non-steroidal anti-inflammatory drug therapy: benefits. Scand J Rheumatol Suppl1996;105:5-12. [ Links ]

15 Seymour, RA; Heasman, PA. Drugs and the periodontium. J Clin Periodontol 1988;15:1-16. [ Links ]

16 Laureano Filho, JR; Maurette, PE; Allais, M; Cotinho, M; Fernandes, C. Clinical comparative study of the effectiveness of two dosages of dexamethasone to control postoperative swelling, trismus and pain after the surgical extraction of mandibular impacted third molars. Med Oral Patol Oral Cir Bucal 2008;13:129-132. [ Links ]

17 Pilatti, GL; André dos Santos, F; Bianchi, A; Cavassim, R; Tozetto, CW. The use of celecoxib and dexamethasone for the prevention and control of postoperative pain after periodontal surgery. J Periodontol 2006;77:1809-1814. [ Links ]

18 Peres, MF; Ribeiro, FV; Ruiz, KG; Nociti-Jr, FH; Sallum, EA; Casati, MZ. Steroidal and nonsteroidal cyclooxygenase-2 inhibitor anti-inflammatory drugs as pre-emptive medication in patients undergoing periodontal surgery. Braz Dent J 2012;23:621-628. [ Links ] 
19 PD, J Jr. A classification of marginal tissue recession. Int J Periodontics Restorative Dent 1985;5:8-13. [ Links ]

20 Ainamo, J; Bay, I. Problems and proposals for recording gingivitis and plaque. Int Dent J 1975;25:229-235. [ Links ]

21 Muhlemann, HR; Son, S. Gingival sulcus bleeding-a leading symptom in initial gingivitis. Helv Odontol Acta 1971;15:107-113. [ Links ]

22 Corah, NL. Development of a dental anxiety scale. J Dent Res 1969;48:596. [ Links ]

23 Jensen, MP; Karoly, P; Braver, S. The measurement of clinical pain intensity: a comparison of six methods. Pain1986;27:117-126. [ Links ]

24 Kolbe, MF; Ribeiro, FV; Luchesi, VH; Casarin, RC; Sallum, EA; Nociti FH Jr; Ambrosano, GM; Cirano, FR; Pimentel, SP; Casati, MZ. Photodynamic therapy during supportive periodontal care: clinical, microbiologic, immunoinflammatory, and patient-centred performance in a split-mouth randomised clinical trial. J Periodontol 2014;85:e277-e86. [ Links ]

25 Steffens, JP; Santos, FA; Sartori, R; Pilatti, GL. Preemptive dexamethasone and etoricoxib for pain and discomfort prevention after periodontal surgery: a double-masked, crossover, controlled clinical trial. J Periodontol 2010;81:1153-1160. [ Links ]

26 Mehra, P; Reebye, U; Nadershah, M; Cottrell, D. Efficacy of anti-inflammatory drugs in third molar surgery: a randomised clinical Trial. Int J Oral Maxillofac Surg 2013;42:835-842. [ Links ]

27 Simone, JL; Jorge, WA; Horliana, AC; Canava,I TG; Tortamano, IP. Comparative analysis of pre-emptive analgesic effect of dexamethasone and diclofenac following third molar surgery. Brazilian oral research 2013;27:266-271. [ Links ]

28 Montgomery, MT; Hogg, JP; Roberts, DL; Redding, SW. The use of glucocorticosteroids to lessen the inflammatory sequelae following third molar surgery. J Oral Maxillofac Surg1990;48:179-187. [ Links ]

29 Czock, D; Keller, F; Rasche, FM; Häussler, U. Pharmacokinetics and pharmacodynamics of systemically administered glucocorticoids. Clin Pharmacokinet 2005;44:61-98. [ Links ]

30 Alcântara, CE; Falci SG; Oliveira-Ferreira, F; Santos, CR; Pinheiro, ML. Pre-emptive effect of dexamethasone and methylprednisolone on pain, swelling, and trismus after third molar surgery: a split-mouth randomised triple-blind clinical trial. Int J Oral Maxillofac Surg2014;43:93-98. [ Links ]

31 Philstrom, BL; Hargreaves, KM; Bouwsma, OJ; Myers, WR; Goodale, MB; Doyle, MJ. Pain after periodontal scaling and root planing. J Am Dent Assoc 1999;130:801-807. [ Links ] 
32 Curtis, JW Jr; McLain, JB; Hutchinson, RA. The incidence of complications and pain following periodontal surgery. J Periodontol 1985;56:597-601. [ Links ]

33 Bystedt, $\mathrm{H}$; Nordenram, A. Effect of methylprednisolone on complications after removal of impacted mandibular third molars. Swed Dent J 1985;9:65-69. [ Links ]

34 Giorgetti, Ana Paula Oliveira, et al. "Preemptive and Postoperative Medication Protocols for Root Coverage Combined with Connective Tissue Graft." Brazilian dental journal 29.1 (2018): 23-29.

(c) (7)

This work is licensed under a Creative Commons Attribution 4.0 International License. 